\title{
A Novel Exact Heuristic Graph Coloring Algorithm based on Finding Independent Set
}

\author{
Sukrati Agrawal \\ M.Tech. Scholar CS Dept. \\ MIST, Indore (M.P.), India
}

\author{
Vishal Chhabra \\ Asst. Prof. CS Dept. \\ MIST, Indore (M.P.), India
}

\begin{abstract}
Vertex coloring is a graph coloring technique which has a wide application area to provide solution for many real world problems. The high computational complexity of graph coloring algorithm led the development of exact heuristic algorithm which can be executed in optimal time. This paper explores some existing graph coloring algorithms to propose taxonomy of exact graph coloring algorithm which is capable to execute large graphs also. This paper presented experimental result on DIMACS graph instances.
\end{abstract}

\section{Keywords}

Graph Coloring, independent set, exact algorithm, approximate algorithm, sequential algorithm and parallel algorithms.

\section{INTRODUCTION}

A graph $\mathrm{G}(\mathrm{V}, \mathrm{E})$ is a set of vertices $\mathrm{V}$ and a set of edges $\mathrm{E}$. The edges are unordered pairs of the form $(i, j)$ where $i, j \in$ $V$. Two vertices $i$ and $j$ are said to be adjacent if and only if $(i, j) \in E$ and non-adjacent otherwise.

The graph coloring problem (GCP) - Graph coloring (mainly vertex coloring) of a graph is an assignment of colors to the vertices such that no two adjacent vertices are assigned the same color. Alternatively, a coloring is a partition of the vertex set into a collection of vertex-disjoint independent sets. Each independent set is called a color class. The GCP is then to find a vertex coloring for a graph using the minimum number of possible colors.

There are numerous applications of graph coloring like time tabling and scheduling [1], register allocation [2], frequency assignments [3], crow management, air traffic management [4] etc. The graph coloring begins with coloring the map. In 1852 Francis Guthrie, while trying to color the map of countries of England, noticed that four colors are sufficed. Subsequently, he proposed that 4 colors are enough to color any map. Successive efforts made to prove Guthrie's 4-color inference led to the development of much of graph coloring. Later on this map coloring is also known as face coloring.

\section{EXISTING ALGORITHMS}

Graph coloring has wide scope of problem solving capabilities. So many researchers and mathematicians tried to discover different algorithms. On the basis of problem solving capabilities graph coloring algorithms are divided into two categories, one is exact and another one is approximate algorithm [5].

\subsection{Exact and Approximate Algorithm}

Finding optimum solution through exact algorithm is a NPhard problem. Exact algorithms give very precious results. But there are certain issues related to exact algorithms are observed. The main problem with the exact algorithms is that most of the exact algorithms are able to execute small size graphs which have less than 100 vertices [6] [7].

Approximate algorithms [5] are algorithms used to find approximate solutions for optimization problems. Heuristic parallel algorithms are the approximate algorithms which color the vertices of the graph in parallel to reduce the coloring time and also reduce the number of colors used in coloring of graph. Solution given by the approximate algorithms is not surely precious. But it has been observed that approximate algorithms are able to provide solution for large graphs having more than 100 vertices.

\subsection{Sequential and Parallel Algorithms}

On the basis of execution behavior algorithms are also divided in to sequential and parallel [8]. Sequential algorithms use to color vertices with minimum colors. These algorithms are much efficient for small size graphs but take time to execute large graphs. There are certain algorithms which uses sequential method to solve the graph coloring such as sequential greedy algorithm [9], first fit [10], largestdegree-first-ordering [11], incidence-degree-ordering [12], and saturation-degree-ordering [13].

Parallel computing is an effective way to find a solution in optimal time complexity for any algorithm. Hence in the same way graph coloring researchers are also using parallel computing to solve GCP. There are some sequential algorithms like largest- degree-first algorithm [11] and the smallest-degree-last algorithm [14] which converted in to parallel algorithms to achieve better performance.

\section{PROBLEM IDENTIFICATION}

By reviewing of literatures related to the graph coloring, it has been found that most of the researcher's focus is to find better chromatic number to solve vertex coloring problem i.e. finding optimum chromatic number is always a primary objective for researchers. Exact algorithm is more reliable for solving GCP. Because result generated by exact algorithms are more accurate and optimum, but the major problem with the existing exact algorithms that they are not suitable to execute large graphs. So it is a real challenge to find such an algorithm, which can execute large graphs in finite time.

\section{PROPOSED SOLUTION}

This paper presents an exact graph coloring algorithm to solve GCP, which can find coloring sequence and feasible chromatic number for large graphs also. Proposed algorithm is based on theory of finding independent set, because all the vertices of any independent set can be colored with same color. Proposed algorithm uses iterative approach to find maximal independent sets to assign colors to the vertices. 


\subsection{Maximal Independent Set}

In graph theory, an independent set is a set of vertices in a graph such that no two vertices are adjacent from given set. That is, it is a set $\mathrm{V}$ of vertices such that for every two vertices in $\mathrm{V}$, there is no dedicated edge connecting the two vertices. This is also known as stable set. If this set is maximal i.e. contain maximal vertices as compared to other sets then it is known as maximal independent set.

\subsection{Proposed Algorithm Flow Chart}

This paper presents an efficient way of finding independent set from a given graph. The algorithm takes graph as an input and generates chromatic no. as an output. The algorithm works in iteration followed by multiple sub iterations. In external iteration the vertex with the maximum degree is selected to process first then this vertex further process by the sub iterations. The algorithm can be better understood by the flow chart given in figure 1 .

\subsubsection{Step 1}

Initially the data set has been initialized for which algorithm reads the graph data such as number of vertices, number of edges and edges information. This data set obtained from the DIMACS graph instance, which is available in .col format. Then algorithm creates an edge set through read edges information. One empty edge cover vertex set is also created to keep the record of vertex information.

\subsubsection{Step 2}

In this step algorithm checks the availability of vertex in vertex set. This condition is checked in all iteration whenever process of finding any independent set has been started. If all vertices are removed from vertex set then algorithm will terminate, otherwise enter into next step.

\subsubsection{Step 3}

In this step first of all copy of vertex set and edge set generated in step 1 is created in order to keep the record of original graph. Edge cover set is also need to clear for this step. After that degree of all vertices available in vertex set is calculated.

\subsubsection{Step 4}

In this step copy of edge set created in previous state is checked, that edge set is empty or not. Empty edge set shows that internal iterations of main iteration (used to find independent set) is over and control goes to the step 6. But if this edge set contain any edge shows internal iterations are not finished and controls transfer to step 5 .

\subsubsection{Step 5}

In this step algorithm finds the vertex having maximum degree from vertex set (calculated in step 3). After finding maximum degree vertex algorithm add this vertex to edge cover set and delete it from vertex set copy (created in step 3). Algorithm also deletes all the edges connected this vertex from the copy of edge set (created in step 3). Finally maximum degree vertex is removed from vertex degree set. Then execution control go to step 4 and repeat step 4 and step 5 till edge set is not empty.

\subsubsection{Step 6}

This step assigns the same color to all the vertices present in a single independent set generated by algorithm. In each iteration algorithm take a new color to assign new independent set. After assigning the color to independent set controls goes to step 2 and whole iteration get repeated till all vertices is not removed from the edge set.

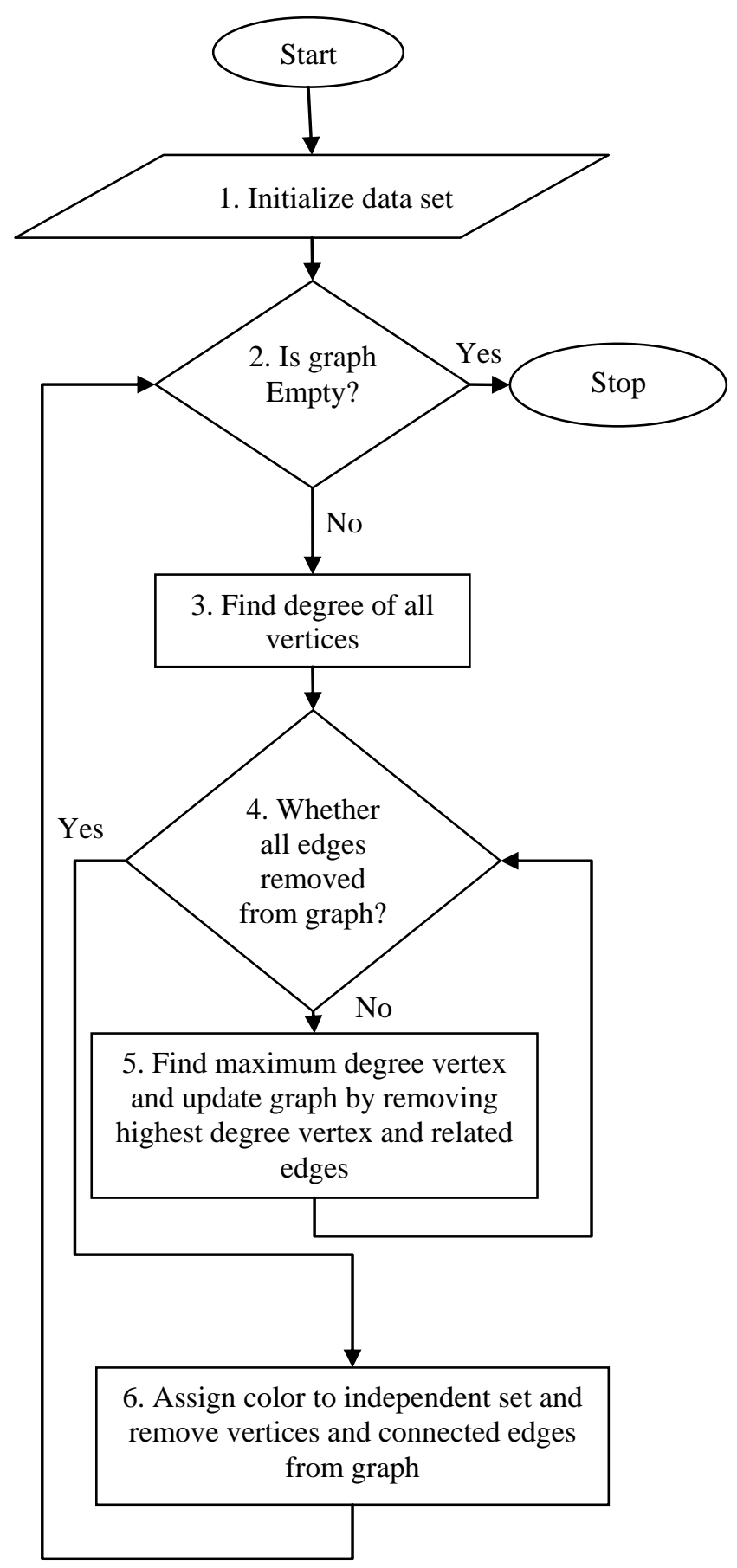

Fig 1: Algorithm Flow Chart

\section{EXPERIMENTAL RESULTS AND RESULT ANALYSIS}

\subsection{Development Environment}

Proposed algorithm is developed in Java Programming Language for experiments. Some important concepts like file handling and collection framework is used to implement the algorithm. Due to the platform independence of java algorithm is can be execute on different operating systems like Windows and Linux. 


\subsection{Data Set}

The Center for Discrete Mathematics and Theoretical Computer Science (DIMACS) is collaboration between Rutgers University, Princeton University, and the research firms AT\&T, Bell Labs, Applied Communication Sciences, and NEC. It is founded to support research activity and provide different open source data sets for research.

DIMACS also provides the different applications and randomly generated graph data sets for graph coloring. Most of the researchers use these data sources for their research. So to experiment the proposed algorithm graph instances provided by DIMACS is used [15].

\subsection{Experimental Results}

To execute the proposed algorithm Intel Pentium Dual CPU G640@2.80 GHz processor and 2.00 GB RAM computer system is used. Table 1 shows the experimental results of proposed algorithm. Table 1 contain following information from first to sixth column serial number (Sr. No.), name of graph Instance (Instance), number of vertices in graph (V), number of edges in graph $(\mathrm{E})$, chromatic number generated by proposed algorithm $(\mathrm{K})$ and execution time in seconds (Time (s)).

Table 1: Experimental results of the proposed algorithm

\begin{tabular}{|c|c|c|c|c|c|}
\hline Sr. No. & Instance & $\mathbf{V}$ & $\mathbf{E}$ & $\mathbf{K}$ & Time (s) \\
\hline 1 & myciel3 & 11 & 20 & 4 & 0.031 \\
\hline 2 & GEOM 20 & 20 & 40 & 5 & 0.031 \\
\hline 3 & myciel4 & 23 & 71 & 5 & 0.047 \\
\hline 4 & queen5_5 & 25 & 320 & 7 & 0.094 \\
\hline 5 & 1-FullIns_3 & 30 & 100 & 4 & 0.047 \\
\hline 6 & GEOM30 & 30 & 80 & 6 & 0.044 \\
\hline 7 & GEOM30a & 30 & 111 & 7 & 0.046 \\
\hline 8 & GEOM30b & 30 & 111 & 6 & 0.062 \\
\hline 9 & queen6_6 & 36 & 580 & 10 & 0.171 \\
\hline 10 & 2-Insertions_3 & 37 & 72 & 4 & 0.042 \\
\hline 11 & GEOM40 & 40 & 118 & 6 & 0.078 \\
\hline 12 & GEOM40b & 40 & 197 & 7 & 0.094 \\
\hline 13 & myciel5 & 47 & 236 & 6 & 0.094 \\
\hline 14 & queen7_7 & 49 & 952 & 12 & 0.25 \\
\hline 15 & GEOM50 & 50 & 177 & 6 & 0.081 \\
\hline 16 & GEOM50b & 50 & 299 & 10 & 0.142 \\
\hline 17 & R50_1g & 50 & 108 & 5 & 0.065 \\
\hline 18 & R50_1gb & 50 & 108 & 5 & 0.075 \\
\hline 19 & R50_5g & 50 & 612 & 15 & 0.218 \\
\hline 20 & $\mathrm{R} 50 \_5 \mathrm{gb}$ & 50 & 612 & 15 & 0.218 \\
\hline 21 & R50_9g & 50 & 1092 & 25 & 0.391 \\
\hline 22 & R50_9gb & 50 & 1092 & 25 & 0.391 \\
\hline 23 & 2-FullIns_3 & 52 & 201 & 5 & 0.078 \\
\hline 24 & 3-Insertions_3 & 56 & 110 & 4 & 0.062 \\
\hline 25 & GEOM60 & 60 & 245 & 7 & 0.125 \\
\hline
\end{tabular}

\begin{tabular}{|c|c|c|c|c|c|}
\hline 26 & GEOM60b & 60 & 426 & 12 & 0.203 \\
\hline 27 & queen8_8 & 64 & 1456 & 14 & 0.329 \\
\hline 28 & 1-Insertions_4 & 67 & 232 & 5 & 0.094 \\
\hline 29 & GEOM70 & 70 & 337 & 9 & 0.174 \\
\hline 30 & GEOM70a & 70 & 529 & 12 & 0.25 \\
\hline 31 & GEOM70b & 70 & 558 & 12 & 0.172 \\
\hline 32 & R75_1g & 70 & 251 & 6 & 0.125 \\
\hline 33 & $\mathrm{R} 75 \_1 \mathrm{gb}$ & 70 & 251 & 6 & 0.125 \\
\hline 34 & Huck & 74 & 602 & 11 & 0.218 \\
\hline 35 & R75_5g & 75 & 1407 & 16 & 0.391 \\
\hline 36 & $\mathrm{R} 75 \_5 \mathrm{gb}$ & 75 & 1407 & 16 & 0.359 \\
\hline 37 & R75_9g & 75 & 2513 & 39 & 0.703 \\
\hline 38 & R75_9gb & 75 & 2513 & 39 & 0.734 \\
\hline 39 & 4-Insertions_3 & 79 & 156 & 4 & 0.094 \\
\hline 40 & 3-FullIns_3 & 80 & 346 & 6 & 0.125 \\
\hline 41 & GEOM80 & 80 & 429 & 8 & 0.206 \\
\hline 42 & Jean & 80 & 508 & 10 & 0.196 \\
\hline 43 & queen9_9 & 81 & 2112 & 15 & 0.375 \\
\hline 44 & David & 87 & 812 & 12 & 0.235 \\
\hline 45 & mug88_1 & 88 & 146 & 5 & 0.109 \\
\hline 46 & mug88_25 & 88 & 146 & 4 & 0.109 \\
\hline 47 & GEOM90 & 90 & 531 & 10 & 0.246 \\
\hline 48 & GEOM90a & 90 & 879 & 16 & 0.36 \\
\hline 49 & GEOM90b & 90 & 950 & 18 & 0.36 \\
\hline 50 & 1-FullIns_4 & 93 & 593 & 5 & 0.172 \\
\hline 51 & myciel6 & 95 & 755 & 7 & 0.219 \\
\hline 52 & queen8_12 & 96 & 2736 & 15 & 0.516 \\
\hline 53 & GEOM100 & 100 & 647 & 10 & 0.282 \\
\hline 54 & GEOM100a & 100 & 1092 & 16 & 0.407 \\
\hline 55 & mug100_1 & 100 & 166 & 4 & 0.125 \\
\hline 56 & mug100_25 & 100 & 166 & 4 & 0.125 \\
\hline 57 & queen10_10 & 100 & 2940 & 17 & 0.5 \\
\hline 58 & R100_1g & 100 & 509 & 8 & 0.235 \\
\hline 59 & R100_5g & 100 & 2456 & 22 & 0.578 \\
\hline 60 & R100_9g & 100 & 4438 & 44 & 1.08 \\
\hline 61 & R100_9gb & 100 & 4438 & 44 & 1.063 \\
\hline 62 & GEOM110 & 110 & 748 & 11 & 0.313 \\
\hline 63 & 4-FullIns_3 & 114 & 541 & 8 & 0.187 \\
\hline 64 & games120 & 120 & 1276 & 9 & 0.344 \\
\hline 65 & GEOM120 & 120 & 893 & 11 & 0.375 \\
\hline 66 & queen11_11 & 121 & 3960 & 18 & 0.703 \\
\hline 67 & DSJC125.1 & 125 & 736 & 8 & 0.284 \\
\hline 68 & DSJC125.5 & 125 & 3891 & 25 & 0.797 \\
\hline 69 & DSJC125.9 & 125 & 6961 & 56 & 1.739 \\
\hline
\end{tabular}




\begin{tabular}{|c|c|c|c|c|c|}
\hline 70 & miles 1000 & 128 & 6432 & 51 & 1.406 \\
\hline 71 & miles 1500 & 128 & 10396 & 81 & 2.588 \\
\hline 72 & miles 250 & 128 & 774 & 10 & 0.297 \\
\hline 73 & miles500 & 128 & 2340 & 26 & 0.531 \\
\hline 74 & miles 750 & 128 & 4226 & 39 & 0.953 \\
\hline 75 & Anna & 138 & 986 & 12 & 0.281 \\
\hline 76 & queen12_12 & 144 & 5192 & 19 & 0.859 \\
\hline 77 & 2-Insertions_4 & 149 & 541 & 5 & 0.203 \\
\hline 78 & 5-FullIns_3 & 154 & 792 & 8 & 0.25 \\
\hline 79 & queen13_13 & 169 & 6656 & 20 & 1.046 \\
\hline 80 & mulsol.i.3 & 184 & 3916 & 31 & 0.719 \\
\hline 81 & mulsol.i.4 & 185 & 3946 & 31 & 0.715 \\
\hline 82 & mulsol.i.5 & 186 & 3973 & 31 & 0.672 \\
\hline 83 & mulsol.i.2 & 188 & 3885 & 31 & 0.672 \\
\hline 84 & myciel7 & 191 & 2360 & 10 & 0.422 \\
\hline 85 & queen14_14 & 196 & 8372 & 21 & 1.375 \\
\hline 86 & mulsol.i.1 & 197 & 3925 & 49 & 1.047 \\
\hline 87 & 1-Insertions_5 & 202 & 1227 & 6 & 0.312 \\
\hline 88 & zeroin.i.3 & 206 & 3540 & 32 & 0.657 \\
\hline 89 & zeroin.i.1 & 211 & 4100 & 51 & 1.04 \\
\hline 90 & zeroin.i.2 & 211 & 3541 & 32 & 0.641 \\
\hline 91 & 2-FullIns_4 & 212 & 1621 & 6 & 0.344 \\
\hline 92 & queen15_15 & 225 & 10360 & 25 & 1.86 \\
\hline 93 & DSJC250.1 & 250 & 3218 & 12 & 0.735 \\
\hline 94 & DSJC250.5 & 250 & 15668 & 42 & 3.578 \\
\hline 95 & DSJC250.9 & 250 & 27897 & 94 & 13.932 \\
\hline 96 & $\mathrm{r} 250.5$ & 250 & 14849 & 101 & 7.327 \\
\hline 97 & queen16_16 & 256 & 12640 & 27 & 2.221 \\
\hline 98 & 3-Insertions_4 & 281 & 1046 & 5 & 0.312 \\
\hline 99 & 1-FullIns_5 & 282 & 3247 & 6 & 0.469 \\
\hline 100 & flat300_28_0 & 300 & 21695 & 45 & 5.954 \\
\hline
\end{tabular}

\section{CONCLUSION}

This paper presents algorithm is to solve vertex coloring problems which can be executed on different types of application data i.e. algorithm supports various application problems. The algorithm is successfully executed up to 300 vertices and gives results in finite time. Execution success rate is also high for proposed algorithm. In some cases it is found that proposed algorithm is not giving optimum chromatic number, so in future algorithm can be update to find optimum chromatic number for all types of graphs.

\section{REFERENCES}

[1] Hussin B., Basari A. S. H., Shibghatullah A. S. and Asmai S. A., Exam Timetabling Using Graph Colouring
Approach, In Proc. IEEE Conference on Open Systems, Langkawi, 25-28 September (2011), p.139-144.

[2] Chaitin G. J., Register Allocation \& Spilling via Graph Coloring, In Proc. SIGPLAN '82 Proceedings of the 1982 SIGPLAN symposium on Compiler construction, June (1982), p.98-105.

[3] S. Ahmed, "Applications of Graph Coloring in Modern Computer Science", International Journal of Computer and Information Technology, 2012, Vol. 3, Issue 2, pp. $1-7$.

[4] Barnier N. and Brisset P., "Graph Coloring for Air Traffic Flow Management", Annals of Operations Research, 130 (1-4), 163-178, August (2004).

[5] A. Gupta and H. Patidar, "A Survey on Heuristic Graph Coloring Algorithm", International Journal for Scientific Research \& Development Vol. 4, Issue 04, 2016, pp. 297-301.

[6] Mahmoudia S., Lotfi S., "Modified Cuckoo Optimization Algorithm (MCOA) to Solve Graph Coloring Problem", Applied Soft Computing, 33, 48-64 (2015).

[7] Torkestani J. A., Meybodi M.R., "A cellular learning automata-based algorithm for solving the vertex coloring problem", Expert Systems with Applications, 38, 9237-9247, (2011).

[8] Patidar H., Chakrabarti P., " Sequential and Parallel Approaches in Context to Graph Coloring Problems - A Survey", International Journal of Computer Systems, Volume 03- Issue 05, May, 201, pp. 403-406.

[9] Allwright JR, Bordawekar R, Codington PD, Dincer K, Martin CL,"A comparison of parallel graph coloring algorithms Technical" Report SCCS-666, Northeast Parallel Architecture Center, Syracuse University, 1995.

[10] Dr. Hussein Al-Omari and KhairEddinSabri: "New Graph Coloring Algorithms", American Journal of Mathematics and Statistics 2 (4): 739-741, 2006ISSN 1549-3636, March 2006.

[11] C. Avanthay, A. Hertz, N. Zufferey, "A variable neighborhood search for graph coloring", European Journal of Operational Research 151 (2) (2003) 379388.

[12] E.K. Burke, B. McCollum, A. Meisels, S. Petrovic, R. $\mathrm{Qu}$, "A graph-based hyper heuristic for timetabling problems", European Journal of Operational Research 176 (2007) 177-192.

[13] E. Falkenauer, "A hybrid grouping genetic algorithm for been packing”, Journal of Heuristics 2 (1) (1996) 5-30.

[14] D. W. Matula and L. L. Beck. "Smallest-last ordering and clustering and graph coloring algorithms", JACM, 1983.

[15] DIMACS Graph Instances, available at "http://mat.gsia.cmu.edu/COLOR/instances.html" 